\title{
Construction and Application of Marine Oil Spill Gravity Vector Differences Detection Model
}

\author{
Weiguang Su${ }^{1,3,4}$, Bo Ping ${ }^{2}$, and Fenzhen $\mathrm{Su}^{3, *}$ \\ ${ }^{1}$ Key Laboratory of Coastal Zone Environmental Processes, Yantai Institute of Coastal Zone \\ Research(YIC), Chinese Academy of Sciences(CAS), Shandong Provincial Key Laboratory of \\ Coastal Zone Environmental Processes, YICCAS, Yantai Shandong China \\ suwg@lreis.ac.cn \\ ${ }^{2}$ School of Remote Sensing and Information Engineering, Wuhan University, Wuhan China \\ pingbalreis.ac.cn \\ ${ }^{3}$ LREIS, Institute of Geographic Sciences and Natural Resources Research, CAS, Beijing China \\ \{suwg, pingb, sufz\} @lreis.ac.cn \\ ${ }^{4}$ University of Chinese Academy of Science
}

\begin{abstract}
This paper proposes a new marine oil spill gravity vector differences detection model based on scalability or viscosity of the oil and water. The model used the median filtering, zero pixels elimination, image normalization, nonlinear transformation, and brought in the law of gravity. The research was upon two oil spill incidents which occurred on the Mediterranean Sea in 2004 and the Gulf of Mexico in 2006. Based on the MODIS remote sensing data, we executed the model to detect the two incidents and compared the results with the results of Sobel detection algorithm. The experimental results illustrated that the model introduced in this paper is superior to Sobel detection algorithm. The proposed model is powerful in oil spill detection.
\end{abstract}

Keywords: marine oil spill, gravity, vector differences.

\section{Introduction}

In recent years, the oil spill pollution accidents caused by wreck on rocks, mechanical breakdown, offshore oil platform leak and pipeline rupture occurred at a frequent rate. These accidents lead to huge ocean pollution, and more seriously, the production and life of the coastal residents are badly harmed. Countries in the world have invested a lot of manpower, materials and financial resources to deal with the various disasters caused by oil spill. How to quickly and accurately discover oil spill has become the most important research subject. The satellite remote sensing is swift, easily synchronized and time-effective, etc., thus it has become an important technique for marine oil spill detection.

Currently, the primary oil spill monitoring sensor comprises a SAR of microwave sensor, and visible / near infrared data of optical sensor. Microwave remote sensing can

\footnotetext{
* Corresponding author.
} 
be in all-weather and all-time operation and this advantage makes it widely applied to the oil spill detection area. SAR image based oil spill detection algorithms are mainly threshold method [1-5], neural networks [6], texture analysis [7-8], wavelet analysis[9-10]. But SAR in the high wind speed region $(>6 \mathrm{~m} / \mathrm{s})$ and low wind speed region $(<1.5 \mathrm{~m} / \mathrm{s})$ [11], and in some natural conditions is prone to produce look-alikes, which increases the difficulty in detecting oil spill. The SAR revisit period is relatively long and uneasy to meet the needs of real-time detection, and its acquisition cost is relatively expensive. In contrast, the optical sensor has a wider range of monitoring, less expensive cost, stronger time-effectiveness and richer spectrum information. It has become an important means of detecting oil spill. An optical remote sensing image based oil spill detection method is founded on the spectrum difference between seawater and the oil film and the oil film characteristics on the images such as band-combination and band-comparison detection[12-13], spectral analysis detection[14], SVM classification detection[15], etc.. However, the contrast of the oil slick and the water is often small, which reduces accuracy of the oil spill detection.

Edge detection as a low-level visual processing, has always been a hot research subject in image processing and analysis area, and many scholars tend to apple edge detection algorithm to detect oil spill[16-18]. However, the above oil spill edge detection algorithms were mainly carried out based on SAR images, as for the optical images, Jing et al. utilized the global minimum active contour model to detect red image spill edge but found when the background is complex, it will detect a false target; additionally the algorithm is less efficient [19]. In recent years, the gravity based image recognition, classification and research aroused many scholars' attention [20-21], but had not been applied to spill detection. This paper is to illustrate how to construct the oil spill gravity vector difference detection model based upon optical remote sensing images, utilizing the expansion or viscosity of the oil and water (i.e. the most easily expanded or viscous nature of the oil and water boundary pixel), bringing in the law of universal gravitation detection method, improving and optimizing this method by zero pixels elimination, normalization and non-linear transformation to the images.

\section{Marine Oil Spill Gravity Vector Differences Detection Model}

\subsection{Gravity Vector Differences Detection Model Theory}

The sea is divided into two parts: oil and water, when marine oil spill occurs. Oil and water have the property of self-expansion or viscosity and can change the properties of the adjacent pixel. So we define the properties as "gravity." On the sea, the expansion of oil is strong, and the expansion of water to oil is weak so that we could assume that oil or water pixel value determines the size of gravity or every pixel is an object and the value of pixel is its "mass". Then, according to Newton's law of universal gravitation, every object in the universe attracts every other object with a force directed along the line of centers for the two objects, and this force will be applied to the two objects respectively, which are equal and in the opposite direction. The law of gravity can be stated [20]: 


$$
\vec{f}_{1,2}=\frac{G m_{1} m_{2} \hat{r}_{2,1}}{\left\|\vec{r}_{2,1}\right\|^{2}}=\frac{G m_{1} m_{2} \vec{r}_{2,1}}{\left\|\vec{r}_{2,1}\right\|^{3}}
$$

Where $\vec{f}_{1,2}$ is the force on object 1 due to object $2, G$ is the gravitational constant which can be approximated as $6.67 \times 10^{-11} \mathrm{~N} \cdot \mathrm{m}^{2} / \mathrm{kg}^{2}, m_{1}$ and $m_{2}$ are the masses of the objects 1 and 2, respectively, $\vec{r}_{2,1}=\left\|\vec{r}_{2}-\vec{r}_{1}\right\|$ is the distance between objects 1 and 2, $\hat{r}_{2,1}=\left(\vec{r}_{2}-\vec{r}_{1}\right) /\left\|\vec{r}_{2}-\vec{r}_{1}\right\|$ is the unit vector from object 2 to 1 . Likewise, the vector gravitational force exerted by object 1 on object 2 takes the form $\vec{f}_{2,1}=-\vec{f}_{1,2}$.

We selected one pixel for the center point and chose $3 \times 3$ calculation window to calculate the gravitational forces:

$$
F=\sqrt{\left(F^{x}\right)^{2}+\left(F^{y}\right)^{2}}
$$

Where $F^{x}$ and $F^{\mathrm{y}}$ are the vector sum of all gravitational forces in $\mathrm{x}$ and $\mathrm{y}$ direction.

\subsection{Marine Oil Spill Gravity Vector Differences Detection Model}

The edge detection algorithm based on law of universal gravitation is only for a few regular images, but for marine oil spill image, which has a large noise, irregular edges of oil slick and the information is weak. This detection algorithm ignores the value of the center pixel in the image is zero. And when the selected calculation window slides in different regions, due to the values of pixel in the different window have large difference will cause the force of the center pixel depend on the value of pixel. Therefore, the detection algorithm has been improved and optimized in the model of this paper.

Firstly, we removed image noise through the image filtering. The filtering methods include Lee, Frost, Gamma and median filtering. The main idea of median filtering is to run through the signal pixel by pixel, replacing each pixel with the median of neighboring pixels and making the values of surrounding pixels close to the true value, thereby it can eliminate noise and maintain edge details. Therefore, we selected median filter before the oil spill detection. We used the same filtering method before the Sobel algorithm to improve the contrast of the detection results.

The pixel's gravity is zero when the value of pixel is zero, through the law of gravity formula, which will affect the oil detection. Therefore, the formula (3) is used to calculate the pixel with value zero and then acquire a new value.

$$
D N_{n}=\frac{D N_{0}+\Delta}{1+\Delta}
$$

Where $D N_{0}$ is the original pixel value, $D N_{n}$ is a new value, $\Delta$ is a very small positive number. 
The image normalization is processed for each $3 \times 3$ calculation window to avoid the algorithm depends on the level of original image brightness. Thereafter, the non-linear transformation is processed via the equation (4) to improve the data contrast.

$$
X_{n(i, j)}= \begin{cases}2 X_{o(i, j)}^{2} & , 0 \leq X_{o(i, j)} \leq 0.5 \\ 1-2\left(1-X_{o(i, j)}\right)^{2}, & 0.5 \leq X_{o(i, j)} \leq 1\end{cases}
$$

Where $X_{n(i, j)}$ is the enhanced pixel value, $X_{o(i, j)}$ is the original pixel value.

Finally, the enhanced image is calculated by gravity vector differences detection model to obtain the sum of the gravitational forces of the neighborhood pixels to the center pixels in the $3 \times 3$ window, then the threshold is selected to obtain the oil slick. The threshold is got via cumulative histogram method. When the proportion of the number of edge pixels to the total of pixels is equivalent to $N \%$, the value of pixel $M$ is the threshold. However, $N$ is determined according to the different data in different regions and through multiple experiments. Comparative experiment threshold selection is also based on this method.

The flow of this model is shown in Fig.1. Firstly, median filtering is used to remove the noise of optical remote sensing image and well maintain the features of image edge. The value of pixel which is equivalent to zero is converted to a new pixel value by using formula (3) to improve the accuracy of the model. Then the model reduces the dependence on brightness of the image by formula (4). Finally marine oil spill is detected by the gravity vector differences detection model.

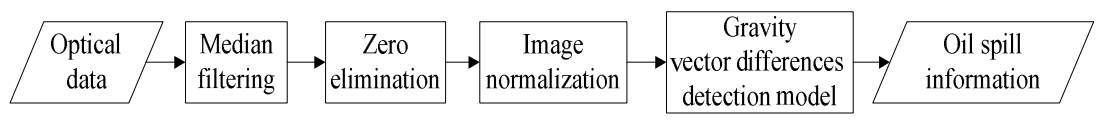

Fig. 1. Flow chart of model

\section{Experimental Data and Result}

\subsection{Data}

This paper takes the oil spill incidents of Mediterranean Sea $\left(33^{\circ}-34^{\circ} \mathrm{N}, 25^{\circ}-27^{\circ} \mathrm{E}\right)$ in 2004 and the Gulf of Mexico $\left(27^{\circ}-28^{\circ} \mathrm{N}, 92^{\circ}-93^{\circ} \mathrm{W}\right)$ in the U.S.A in 2006 as research subjects. The variance of the sea water spectrum is less than the contrast of oil-water spectrum of the second band of MODIS, and the oil slick can be shown on this band clearly [22]. So this paper selects the MODIS data acquired on 30th August 2004 and 13th May 2006 with spatial resolution is 250m to detect the oil. 


\subsection{Result}

Case1:

In late Aug. 2004, the oil spill incident was happened in the Mediterranean Sea, as shown in the Fig.3 (a). Two parts of A and B exhibited linear which complied with the distribution of trajectory of ship oil spill in the sea. The region $\mathrm{C}$ may be the shadow caused by the wind speed or wave which was not oil spill. Oil spill was detected according to the proposed model and Sobel algorithm. A threshold was determined by the cumulative histograms shown in Fig.2. After several tests, we selected gray value as the threshold when $\mathrm{N}=98 \%$. The result of gravity vector difference detection model is shown in Fig. 3 (b). The feature of the oil is linear in area A and boundary is continuous and oil slick is reflected clearly. The overall effect is similar to the actual spill area in area $\mathrm{B}$, but the final oil slick is incomplete. The wrong test result appears in the spill area C. The result of Sobel is shown in Fig.3 (c). The oil slick is missed on the left of area $\mathrm{A}$. The oil of the area $\mathrm{B}$ is complete. Although the area $\mathrm{C}$ is not clear, false detection exists. The noise of the result is prominent.
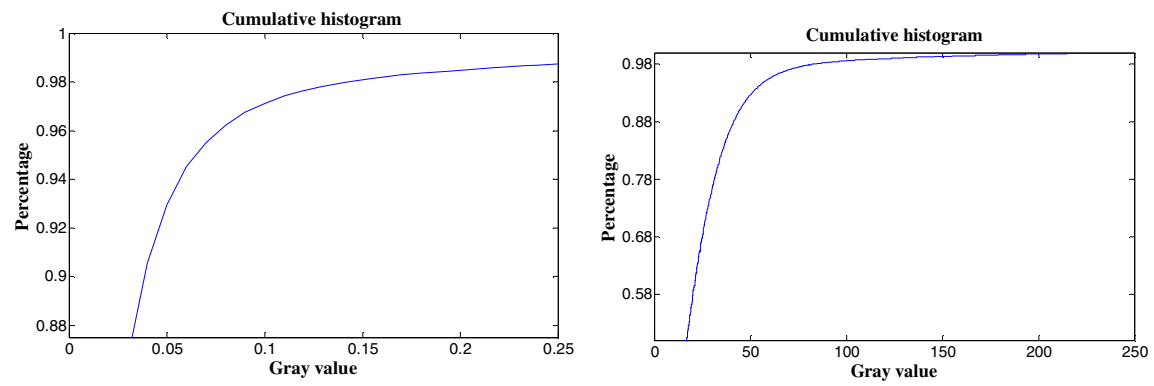

(a) Cumulative histogram of result of proposed model (b) Cumulative histogram of result of Sobel

Fig. 2. Cumulative histograms

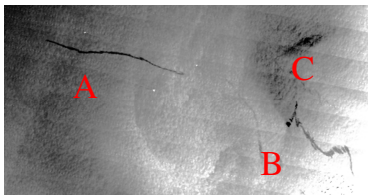

(a) Original image

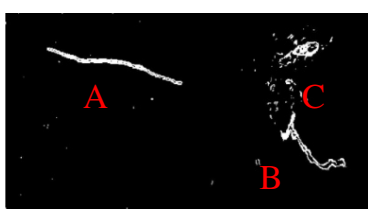

(b) Result of proposed model

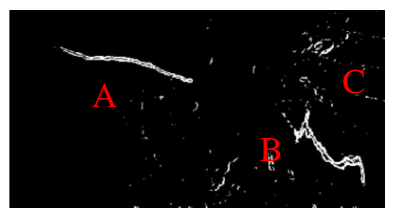

(c) Result of Sobel

Fig. 3. Detection results of oil spill in the Mediterranean Sea in 2004

\section{Case 2:}

The oil spill incident was happened in Gulf of Mexico of the U.S.A in 2006, as shown in the Fig.5 (a). The oil spill affected by sea waves, was "banded" distributed throughout the sea and formed obvious contrast with the background. First the 
cumulative histograms of gravity vector difference detection model and Sobel algorithm were obtained as shown in Fig.4. We selected gray value when $\mathrm{N}=85 \%$ as the threshold after several tests. The result of gravity vector difference detection model is shown in Fig.5 (b). The overall effect is relatively good and oil slick is prominent. Some areas are partially affected by noise so that the final result is impacted. The model misses to detect some "narrow" film and some areas with inconspicuous contrast between the oil and water. The result of Sobel is shown in Figure 5 (c). The right half is badly affected by noise, and only the left part of oil can be detected, but the oil slick is not quite clear due to the noise, and the upper left oil slick is not detected.
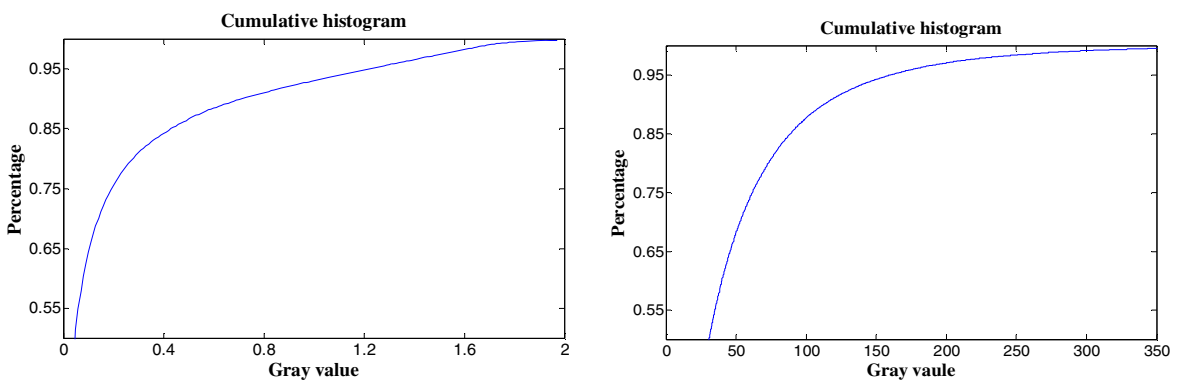

(a) Cumulative histogram of result of proposed model (b) Cumulative histogram of result of Sobel

Fig. 4. Cumulative histograms

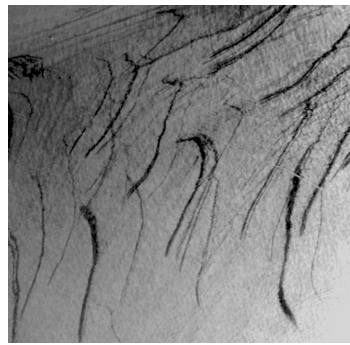

(a) Original image

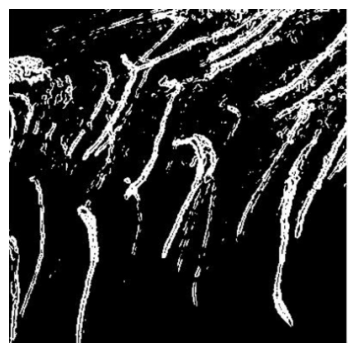

(b) Result of proposed model

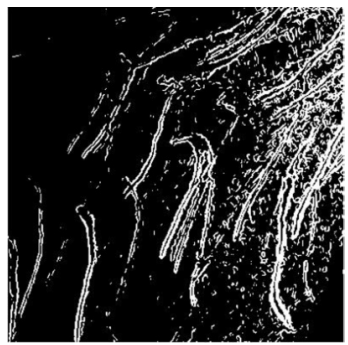

(c) Result of Sobel

Fig. 5. Detection results of oil spill in the Gulf of Mexico in 2006

\section{Discussion}

The detection results of the two cases above prove that the proposed model has a strong capability for oil spill detection. But in some areas, the sea waves result in the area of remote sensing image is in dark shade, forming look-alikes as shown in area C of Fig. 3, which will reduce the accuracy of the model. This paper selects the MODIS 
near-infrared data with the spatial resolution is $250 \mathrm{~m}$ which is relatively low. Some small oil slick is similar to the background in the image, and some new oil is brighter than the earlier oil in the image. Therefore, in the detection process, some oil is undetected due to the contrast between the oil and background is not obvious, as shown in Fig.3 and Fig.5. The accuracy of this model is influenced by sunglint. Sunglint is the direct specular reflection of the solar radiation over the ocean waves. As a result, the typical image of a sea surface portion acquired in sunglint condition is brighter than the surrounding region [23] as shown in area B of Fig.3 (a). Especially at the end of area B, the contrast between oil slick and water is reduced which is caused by the color of oil slick inversion due to the sunglint. This makes the end of oil slick undetected.

\section{Conclusions}

This paper proposes a marine oil spill gravity vector differences detection model based on gravitational forces, the model removed noise from the image using median filtering, and used the zero pixels elimination and the image normalization to avoid the dependency of the model on the brightness level of original data. Then the contrast between oil slick and water was increased by nonlinear transformation. Finally, we calculated the gravity vector between the center pixel and neighborhoods and obtained the oil slick through the judgment against the threshold. We had good results in two test areas. The results proved that the proposed model has a strong capability at marine oil spill detection.

There are many factors affect marine oil spill detection, such as oil slick brightness inversion phenomenon under the sunglint, judgment and detection of suspected oil slick, the threshold selection. These factors will inevitably affect the accuracy of marine oil spill detection. Therefore, in future research, we should select more optical satellite remote sensing data to modify the model, and enhance the accuracy of the model.

Acknowledgments. This research was supported by the National Key Technology R\&D Program of China (Project number: 2011BAH23B04) and the National High Technology Research and Development Program of China (863 Program) (Project number: 2007AA092202).

\section{References}

1. Fiscella, B., Giancaspro, A., Nirchio, F.: Oil Spill Detection Using Marine SAR Images. International Journal of Remote Sensing 21, 3561-3566 (2000)

2. Solberg, A.H.S., Storvik, G., Solberg, R.: Automatic Detection of Oil Spills in ERS SAR Images. IEEE Transactions on Geoscience and Remote Sensing 4, 1916-1924 (1999)

3. Solberg, A.H.S., Brekke, C., Husoy, P.O.: Oil Spill Detection in Radarsat and Envisat SAR Images. IEEE Transactions on Geoscience and Remote Sensing 45, 746-755 (2007)

4. Kanaa, T.F.N., Tonye, E., Mereier, G.: Detection of Oil Slick Signatures in SAR Images by Fusion of Hysteresis Thresholding Responses. In: International Geoscience and Remote Sensing Symposium, vol. 4, pp. 2750-2752 (2003) 
5. Mera, D., Cotos, J.M., Varela-Pet, J.: Adaptive Thresholding Algorithm Based on SAR Images and Wind Data to Segment Oil Spills Along the Northwest Coast of the Iberian Peninsula. Marine Pollution Bulletin 64(10), 2090-2096 (2012)

6. Topouzelis, K., Karathanassi, V., Pavlakis, P.: Detection and Discrimination Between Oil Spills and Look-alike Phenomena Through Neural Networks. ISPRS Journal of Photogrammetry and Remote Sensing 62, 264-270 (2007)

7. Maged, M.: RADARSAT Automatic Algorithms for Detecting Coastal Oil Spill Pollution. International Journal of Applied Earth Observation and Geoinformation 3, 191-196 (2001)

8. Poonam, M.B., Sonali, P.: Oil Spill Detection in SAR Images Using Texture Entropy Algorithm and Mahalanobis Classifier. International Journal of Engineering Science and Technology 4(12), 4823-4826 (2012)

9. Wu, S.Y., Liu, A.K.: Towards an Automated Ocean Feature Detection, Extraction and Classification Scheme for SAR imagery. International Journal of Remote Sensing 5, 935-951 (2003)

10. Derrode, S., Mercier, G.: Unsupervised Multiscale Oil Slick Segmentation From SAR Images Using a Vector HMC Model. Pattern Recognition 40(3), 1135-1147 (2007)

11. Liu, A.K., Wu, S.Y., Tseng, W.Y.: Wavelet Analysis of SAR Images for Coastal Monitoring. Canadian Journal of Remote Sensing 26, 494-500 (2000)

12. Hu, C.M., Li, X.F., William, G.P.: Detection of Natural Oil Slicks in the NW Gulf of Mexico Using MODIS Imagery. Geophysical Research Letters 36(1) (2009)

13. Srivastava, H., Singh, T.P.: Assessment and Development of Algorithms to Detection of Oil Spills Using MODIS Data. J. Indian Soc. Remote Sens. 38, 161-167 (2010)

14. Ma, L., Li, Y., Liu, Y.: Oil Spill Monitoring Based on Its Spectral Characteristics. Environmental Forensics 10(4), 317-323 (2009)

15. Cococcioni, M., Corucci, L., Masini, A.: SVME: An Ensemble of Support Vector Ma-chines for Detecting Oil Spills From Full Resolution MODIS Images. Ocean Dynamics 2, 449-467 (2012)

16. Jubai, A., Jing, B., Yang, J.: Combining Fuzzy Theory and A Genetic Algorithm for Satel-lite Image Edge Detection. International Journal of Remote Sensing 14, 3013-3024 (2005)

17. Karantzalos, K., Argialas, D.: Automatic Detection and Tracking of Oil Spills in SAR Imagery with Level Set Segmentation. International Journal of Remote Sensing 21, 6281-6296 (2008)

18. Jing, Y., Jubai, A., Zhaoxia, L.: Edge Detection Algorithm of Oil Spills Remote Sensing Image Based on DBT Denoising and Improved GDNI Edge Linking. Computer Science 38(11), 282-285 (2011)

19. Jing, Y., Jubai, A., Zhaoxia, L.: A Novel Edge Detection Algorithm Based on Global Minimization Active Contour Model for Oil Slick Infrared Aerial Image. IEEE Transactions on Geoscience and Remote Sensing 49(6), 2005-2013 (2011)

20. Sun, G.Y., Liu, Q.H., Liu, Q.: A Novel Approach for Edge Detection Based on The Theory of Universal Gravity. Pattern Recognition 4, 2766-2775 (2007)

21. Lopez-Molina, C., Bustinc, H., Fernandez, J.: A Gravitational Approach to Edge Detection Based on Triangular Norms. Pattern Recognition 43, 3730-3741 (2010)

22. Su, W., Su, F., Zhou, C.: Optical Satellite Remote Sensing Capabilities Analysis of the Marine Oil Spill. Journal of Geo-Information Science 14(4), 523-530 (2012)

23. Adamo, M., De Carolis, G., De Pasquale, V.: Exploiting Sunglint Signatures From MERIS and MODIS Imagery in Combination to SAR Data to Detect Oil Slicks. In: Envisat Symposium, pp. 23-27 (2007) 